\title{
Analysis of levitation of saturated liquid droplets on permeable surfaces
}

\author{
M. FATEHI and M. KAVIANY \\ Department of Mechanical Engineering and Applied Mechanics, The University of Michigan, \\ Ann Arbor, Mi 48109, U.S.A.
}

(Received 14 February 1989 and in final form 2 August 1989)

\begin{abstract}
Steady-state evaporation of a saturated liquid droplet and its levitation over a solid surface due to momentum-induced pressure in the vapor film is studied by solving the axisymmetric governing equation numerically. A previous one-dimensional analysis shows that the distance (or gap) between the solid surface and the droplet decreases when the vapor can penetrate the solid. For porous layers, this onedimensional analysis which is based on Brinkman's extension to Darcy's law predicts momentum boundary layer thicknesses of the order of the square root of the permeability. In typical porous solids, this thickness is smaller than the pore or particle size. In the present study the Beavers-Joseph semi-empirical boundary condition at the interface of the plain (i.e. vapor underneath the droplet) and permeable layers is used instead of this one-dimensional model. The reduction in the gap size with respect to variations in permeability and thickness of the porous layer is determined for the ranges where this boundary condition is valid. The effect of vapor escape through the bottom surface of the porous layer is also studied. This axisymmetric model predicts an asymptotic value for the slip velocity and the gap size as the permeability increases beyond a certain value. This failure of the model to predict the collapse of the droplet is due to the breakdown of the Beavers-Joseph interfacial condition for high permeabilities. However, for practical applications the surface roughness is expected to dominate when the gap size approaches zero. The onedimensional model, i.e. the Brinkman extension, on the other hand, predicts an unrealistic rapid drop of the gap size when the permeability is increased beyond a certain value, because of the assumption of equal pressure gradients in the porous and plain layers.
\end{abstract}

\section{INTRODUCTION}

LEVITATION of saturated liquid droplets has previously been studied experimentally and analytically by Avedisian and Koplik [1]. In their experimental study they observed a reduction in the droplet evaporation time whenever the solid surface over which the droplets were levitated was permeable. It should be noted that surface roughness can also result in a decrease in the droplet evaporation time due to a reduction of the average vapor film thickness and a consequent increase in the heat transfer rate. In practice, it is difficult to construct porous layers which have surface roughnesses which are significantly smaller than their pore or particle size. Therefore, in these experiments, it is the combined effects of surface roughness and suction that result in higher evaporation rates.

Avedisian and Koplik's [1] analytical study is based on a Brinkman extension of Darcy's law for flow through porous media. This is equivalent to the addition of a macroscopic boundary effect to the otherwise bulk resistance model for the flow. They also used a heuristic interfacial boundary condition between the vapor film and the porous layer, which in principle accounts for the presence of the solid matrix and leads to a balance of the shear stress on the fluid at the interface. The result of their analysis gives a momentum boundary layer thickness just inside the porous layer, which is of the order of the pore size. This finding contradicts the basic assump- tion required for a continuum treatment of the solid and fluid phases by a single momentum equation, i.e. the elementary representative volume encompasses a large enough volume such that an ensemble average over a large number of pores is made and yet important physical features such as the boundary layers are not masked. This shortcoming of the Brinkman extended analysis has gone unnoticed in other reported work such as ref. [2], in which a fully developed momentum boundary layer thickness equal to $(4 K / \varepsilon)^{1 / 2}$ and a development length equal to $K U_{\infty} / v$ were found for forced convection in porous media bounded on one side by a flat plate. Depending on the structure of the porous medium, this boundary layer thickness can be of the order of a tenth of the pore size. Furthermore, without a special coordinate stretching, in numerical solution the mesh size (or the period of the trial functions) have to be of the order of a tenth of this boundary layer in order to successfully resolve the variation of the velocity field within the boundary. This requires resolution of the order of $0.1 K^{1 / 2}$ where the surface roughness is of the order of $10 K^{1 / 2}$ and the linear dimension of the porous medium is of the order of $10^{3} \mathrm{~K}^{1 / 2}$ or larger.

In practice, the permeability of the porous layer near the surface is not uniform and, depending on the manufacturing process, it can be larger near the surface. When coupled with the unavoidable surface roughness, this makes a general analytical treatment of the flow near the interface almost impossible. Direct 


\section{NOMENCLATURE}

$d_{\mathrm{p}} \quad$ particle size [m]

$F$ integral pressure force at the droplet base [N]

g gravitational constant

$h_{1} \quad$ thickness of the porous layer [m]

$h_{2}$ thickness of the plain layer [m]

$h_{\mathrm{lv}} \quad$ latent heat of vaporization $\left[\mathrm{kJ} \mathrm{kg}^{-1}\right]$

$k$ vapor thermal conductivity [ $\left.\mathrm{W} \mathrm{m}^{-1} \mathrm{~K}^{-1}\right]$

$K$ permeability [ $\left.\mathrm{m}^{2}\right]$

$l \quad$ vertical distance above the droplet base [m]

$p \quad$ pressure $\left[\mathrm{N} \mathrm{m}^{-2}\right]$

$p_{0} \quad$ ambient pressure around the droplet [ $\mathrm{N} \mathrm{m}^{-2}$ ]

$r, x$ dimensionless radial and axial coordinate axes, $R / R_{0}, X / R_{0}$

$R_{0} \quad$ radius of droplet [m]

$R, X$ radial and axial coordinate axes $[\mathrm{m}]$

$V_{0} \quad$ axial vapor velocity at the droplet base $\left[\mathrm{m} \mathrm{s}^{-1}\right]$

Re Reynolds number, $R_{0} V_{0} / v$

$T_{p} \quad$ surface temperature at the top of the porous layer [K]

$T_{\text {sat }} \quad$ liquid saturation temperature $[\mathrm{K}]$

$U, V$ radial and vertical velocity in the vapor film $\left[\mathrm{m} \mathrm{s}^{-1}\right]$

$u, v$ dimensionless radial and vertical velocity in the vapor film, $U / V_{0}, V / V_{0}$
$U_{\mathrm{i}} \quad$ radial velocity at the vapor film/porous layer interface $\left[\mathrm{m} \mathrm{s}^{-1}\right]$

$V_{\mathrm{d}} \quad$ droplet volume $\left[\mathrm{m}^{3}\right]$

$W$ droplet weight $[\mathrm{N}]$.

\section{Greek symbols}

$\alpha \quad$ Beavers-Joseph proportionality constant

$\delta \quad$ vapor film thickness [m]

$\Delta \quad$ boundary layer thickness in the porous matrix [m]

$\varepsilon \quad$ porosity

$\mu$ dynamic viscosity in the vapor film $\left[\mathrm{kg} \mathrm{m}^{-1} \mathrm{~s}^{-1}\right]$

$\mu_{\text {eff }} \quad$ effective dynamic viscosity in the porous medium $\left[\mathrm{kg} \mathrm{m}^{-1} \mathrm{~s}^{-1}\right]$

$v \quad$ kinematic viscosity in the vapor film $\left[\mathrm{m}^{2} \mathrm{~s}^{-1}\right]$

$\rho \quad$ vapor density $\left[\mathrm{kg} \mathrm{m}^{-3}\right]$

$\rho_{1} \quad$ liquid density $\left[\mathrm{kg} \mathrm{m}^{-3}\right]$

$\psi \quad$ dimensionless stream function

$\Psi \quad$ stream function $\left[\mathrm{m}^{3} \mathrm{~s}^{-1}\right]$

$\omega$ dimensionless vorticity

$\Omega \quad$ vorticity [ $\left.\mathbf{s}^{-1}\right]$.

\section{Subscripts}

cl center line

D Darcy's

$\infty$ free stream. simulation of the flow adjacent to these types of interfaces may clarify some of the pore-level phenomena [3-5]. Larson and Hingdon $[4,5]$ studied the microscopic flow near the surface of a porous medium consisting of semi-infinite lattices of cylindrical inclusions. They predicted a rapid decay of velocity over a single lattice cell even at high porosities, which in general supports the boundary layer thickness predicted by the Brinkman model. However, they also concluded that Brinkman's equation fails to predict the flow field for transverse flow through anisotropic porous media. In addition, their analysis showed that the slip velocity (similar to the Beavers-Joseph slip velocity) is extremely sensitive to the position of the interface. From their results it appears that the slip velocity model, along with an empirically determined boundary condition, describes the interfacial phenomenon more satisfactorily. A model based on the ensemble average of flow through some interfacial conduits has been proposed by Moaveni-Sabet [6].

Beavers and Joseph [7] obtained a semi-empirical relationship for the interfacial velocity of the flow through a channel bounded on one side by a porous layer; an imposed pressure gradient induces flow through both layers. They observed that the bulk permeability and bulk porosity are inadequate par- ameters for the description of the interfacial velocity. However, with an adjustable constant (that varies over an order of magnitude) they found a relationship for the interfacial velocity. Neale and Nader [8], and by a similar approach Vafai and Thiyagaraja [9], applied the Brinkman extended analysis and showed that this constant is equivalent to an effective viscosity that is used for the presumed continuity of the shear stress at the interface. Nield [10-12] applied the boundary condition developed by Beavers and Joseph. Saffman [13], through dimensional, scaling and boundary-layer (inner and outer solutions) arguments, showed that the constant in the BeaversJoseph correlation depends on where the interface is taken. He shows that within the uncertainty of the order of $10 K^{1 / 2}$ (associated with surface roughness) in the location of the interface, this constant can change several fold.

This study considers the steady-state flow of vapor, which is emitted from the surface of an evaporating droplet, through the vapor film and the permeable solid (which provides the heat for evaporation). Figure 1 gives a schematic of the problem. The axisymmetric flow equations are solved numerically. The Beavers-Joseph boundary condition, which was obtained for one-dimensional flow, is applied to this 


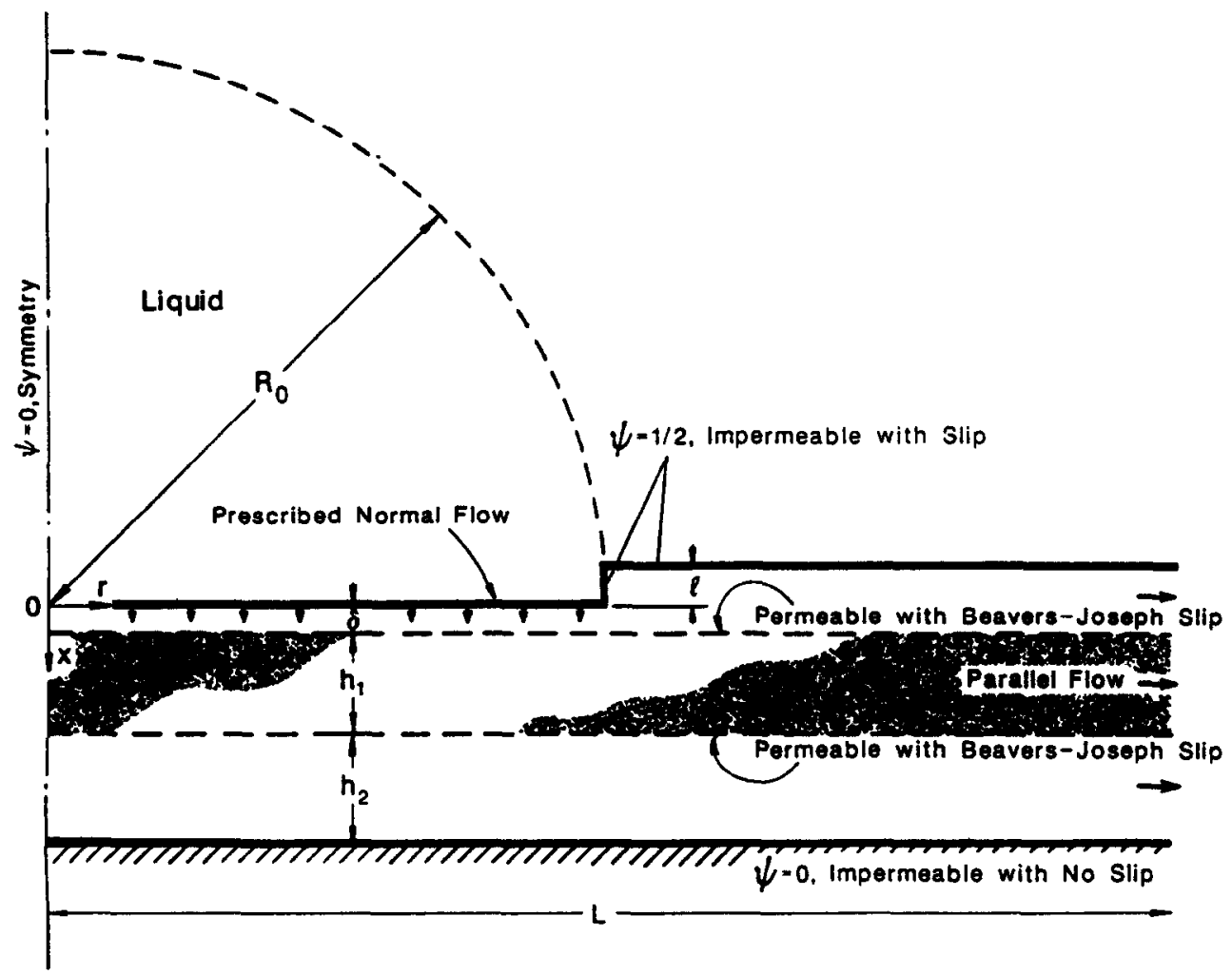

Fig. 1. A schematic of the system and the computational domain.

axisymmetric problem; this extension is assumed to be possible provided the additional component of the plain layer shear stress along the interface is included. (Presently, no experimental or theoretical validation of this assumption is available.) The flow through the porous layer is described by Darcy's law (zero vorticity), and the reduction of the gap size is examined along with the flow through the vapor film and the porous layer. In addition, the effect of flow through the lower boundary of the porous layer into another plain layer is also studied. The heat transfer between the upper surface of the porous layer and the droplet is nearly dominated by conduction because the Peclet number $\delta V_{0} / \alpha$ is rather small (for the examples given here about 0.30 ). Note that only a fraction of the flow enters the porous layer; therefore, the effective Peclet number is even smaller. For the case of a porous layer sandwiched between two plain layers, the heat supply can be through volumetric heat generation in the porous layer.

\section{ANALYSIS}

Since only idealized analytical treatment of levitated droplet evaporation is possible, the following assumptions [1] are made : (i) the evaporation is quasisteady and takes place in the film boiling regime; (ii) the droplet is hemispherical (for a methanol droplet with a radius of $5 \mathrm{~mm}$ the Bond number is less than one); (iii) the droplet is at rest and isothermal at saturation ; (iv) the surface temperature of the porous medium remains constant and exceeds the Leidenfrost temperature of the liquid; (v) the vapor film thickness is uniform and very small, heat transfer is dominated by conduction, and radiation is negligible; (vi) mass transfer from the upper surface of the droplet is neglected; and (vii) the vapor flow in the film is laminar and viscous $(R e \approx 14)$.

The continuity and momentum equations (for axisymmetric, quasi-steady flow) in the vapor film are

$$
\begin{gathered}
\nabla \cdot \mathbf{U}=0 \\
(\mathbf{U} \cdot \nabla) \mathbf{U}=-\rho^{-1} \nabla p+v \nabla^{2} \mathbf{U}
\end{gathered}
$$

and for the porous medium we have

$$
\begin{gathered}
\nabla \cdot \mathrm{U}_{\mathrm{D}}=0 \\
0=-\rho^{-1} \nabla p-K^{-1} v \mathrm{U}_{\mathrm{D}} .
\end{gathered}
$$

Defining the vorticity as

$$
\mathbf{\Omega}=\frac{\partial U}{\partial X}-\frac{\partial V}{\partial R}
$$

and introducing

$$
\begin{gathered}
U=-\frac{1}{R} \frac{\partial \Psi}{\partial X} \\
V=\frac{1}{R} \frac{\partial \Psi}{\partial R}
\end{gathered}
$$


we can write equation (2) in the dimensionless form as

$$
\frac{\partial \omega}{\partial t}+\frac{\partial(u \omega)}{\partial r}+\frac{\partial(v \omega)}{\partial x}=\frac{1}{\operatorname{Re}}\left\{\frac{\partial}{\partial r}\left[\frac{1}{r} \frac{\hat{c}}{\hat{\partial} r}(r \omega)\right]+\frac{\partial^{2} \omega}{\partial x^{2}}\right\}
$$

where $R_{0}, V_{0}$, and $\rho V_{0}^{2}$ are used to scale the length, velocity, and pressure for the dimensionless Reynolds number

$$
R e=\frac{V_{0} R_{0}}{v}
$$

The stream function is related to the vorticity by

$$
-\omega=\frac{1}{r} \frac{\partial^{2} \psi}{\partial x^{2}}+\frac{\partial}{\partial r}\left(\frac{1}{r} \frac{\partial \psi}{\partial r}\right)
$$

The vapor velocity at the base of the droplet, $V_{0}$, is found from the balance of energy at the liquid/vapor interface

$$
V_{0}=\frac{k\left(T_{\mathrm{p}}-T_{\text {sat }}\right)}{\rho h_{\mathrm{lv}} \delta}
$$

This equation remains valid for rather small vapor flows into the porous layer, although for large flows (not attempted here) the contribution of convection (Peclet number) must be included. It is assumed that $T_{\mathrm{p}}$ remains the same despite the suction.

Darcy's law governing the flow in a porous medium (equation (4)) written in the stream function-vorticity formulation reduces to

$$
\omega_{\mathrm{D}}=0
$$

while equations (6), (7), and (9) still hold (zero vorticity in the porous medium indicates that the Reynolds number is less than one). The initial conditions used are

$$
\psi=\omega=0
$$

and the boundary conditions are (also given in Fig. 1)

$$
\begin{gathered}
0 \leqslant r \leqslant 1, x=0, \quad u=0, v=1 \\
\omega=-\frac{1}{r} \psi_{x x}, \psi=\frac{1}{2} r^{2} \\
r=0,0<x<\delta+h_{1}+h_{2}, \quad u=0, v=\frac{1}{r} \psi_{r} \\
\quad \omega=\psi=0 \\
r=1,-l \leqslant x<0, \quad u=0, v=\frac{1}{r} \psi_{r} \\
\quad \omega=\frac{1}{r^{2}} \psi_{r}-\frac{1}{r} \psi_{r r}, \psi=\frac{1}{2} \\
1<r \leqslant 2, x=-l, \quad u=-\frac{1}{r} \psi_{x}, v=0 \\
\omega=-\frac{1}{r} \psi_{x x}, \psi=\frac{1}{2}
\end{gathered}
$$

$$
\begin{aligned}
r=2,-l<x<\delta+h_{1}+h_{2}, \quad u & =-\frac{1}{r} \psi_{x}, v=0 \\
\omega & =-\frac{1}{r} \psi_{x x} \\
0 \leqslant r \leqslant 2, x=\delta+h_{1}+h_{2}, \quad u & =r=\psi=0 \\
\omega & =-\frac{1}{r} \psi_{x x} .
\end{aligned}
$$

The maximum value of the stream function is equal to $\frac{1}{2}$ at the edge of the droplet (where both $R / R_{0}$ and $V / V_{0}$ are equal to 1 ) and remains constant around and above the droplet. $l$ is the height of the computational domain above the droplet base to model the vapor flow around the droplet as it emerges through the vapor layer underneath the droplet. The boundary condition adopted at the interface of the vapor film and the porous surface is the one-dimensional empirical model proposed by Beavers and Joseph [7]

$$
\frac{\mathrm{d} U}{\mathrm{~d} X}=\frac{\alpha}{\sqrt{K}}\left(U_{\mathrm{i}}-U_{\mathrm{D}}\right)
$$

where $\boldsymbol{x}$ is a dimensionless parameter that is a characteristic of the porous layer and is determined experimentally, $U_{\mathrm{i}}$ the radial vapor velocity in the film adjacent to the porous surface, and $L_{D}$ the Darcean velocity given by

$$
U_{\mathrm{D}}=-\frac{K}{\mu} \frac{\mathrm{d} p}{\mathrm{~d} X}
$$

A general form of equation (13) is proposed by Nield [11] as

$$
\frac{\partial U}{\partial X}+\frac{\partial V}{\partial R}=\frac{x}{\sqrt{K}}\left(C_{\mathrm{i}}-\zeta_{\mathrm{D}}\right)
$$

It should be noted that the convective Darcean velocity, $U_{\mathrm{D}}$, in the Beavers-Joseph interfacial model (equation (13)) is due to an applied pressure gradient along the porous layer as well as the plain layer. Since flow through the porous layer is caused by the influx through the interface in the problem here, we modified equation (13) by setting $U_{\mathrm{D}}$ equal to zero in order to produce realistic results (see the Appendix for a detailed explanation). The term $\hat{c} V^{\prime} \hat{c} R$, which was added to the original Beavers-Joseph model because of the extra velocity component, is found to be rather small in magnitude compared to $\vec{C} C \vec{C} X$.

The pressure distribution at the base of the droplet, $p(R)$, can be determined for values of $d^{2} U / d X^{2}$ and $\omega$ evaluated at the base of the droplet by the numerical integration of equation (15):

$$
\int_{p_{\mathrm{c}}}^{p} \mathrm{~d} p=\mu \int_{0}^{R} \frac{\mathrm{d}^{2} U}{\mathrm{~d} X^{2}} \mathrm{~d} R-\left.\rho V_{0}\right|_{0} ^{R} \omega \mathrm{d} R
$$


When the weight of the droplet is balanced by the surface integral of pressure forces, we have

$$
\int_{0}^{R_{n}}\left(p-p_{0}\right) 2 \pi R \mathrm{~d} R=g\left(\rho-\rho_{1}\right) V_{\mathrm{d}}=W .
$$

\section{SOLUTION AND VALIDATION}

The governing equations (6)-(12) subject to the boundary conditions shown in Fig. 1 are solved numerically using the explicit, two-step finite difference scheme and the successive over-relaxation technique described by Jaluria and Torrance [14]. Steadystate solutions are obtained for $\psi, \omega, u$, and $v$ in the vapor film and porous matrix and around the droplet.

Proper mixing of forward and backward finitedifferencing of the interfacial condition is needed in order to properly couple the rotational flow in the plain layer with the irrotational flow in the porous layer. The normalized interfacial condition of equation (14) for $U_{D}=0$ is combined with the continuity of the velocity in the $x$ - and $r$-directions at the interface and then written in the following second order, finitedifference approximation for each grid point $(i, j)$ along the interface :

$$
\begin{aligned}
A \psi_{i, j}=B_{1} \psi_{i, j-3} & +B_{2} \psi_{i, j-2}+B_{3} \psi_{i, j+2} \\
& +B_{4} \psi_{i, j+1}+B_{3} \psi_{i-1, j}+B_{6} \psi_{i+1 . j}
\end{aligned}
$$

where $i$ and $j$ denote the indices in the $r$ - and $x$ directions, respectively. The coefficients of the stream function in equation (17) are given by

$$
\begin{gathered}
A=-\frac{11}{2 \Delta x^{2}}-\frac{3 \alpha R_{0}}{2 \sqrt{ } K \Delta x}-\frac{2}{\Delta r^{2}} \\
B_{1}=\frac{1}{\Delta x^{2}} \\
B_{2}=-\frac{11}{4 \Delta x^{2}} \\
B_{3}=\frac{5}{4 \Delta x^{2}}+\frac{\alpha R_{0}}{2 \sqrt{ } K \Delta x} \\
B_{4}=-\frac{5}{\Delta x^{2}}-\frac{2 \alpha R_{0}}{\sqrt{ } K \Delta x} \\
B_{5}=-\frac{1}{2 r \Delta r}-\frac{1}{\Delta r^{2}} \\
B_{6}=\frac{1}{2 r \Delta r}-\frac{1}{\Delta r^{2}} .
\end{gathered}
$$

Note that for two-dimensional flows, the parallel component of the velocity does not jump over the interface (as is expected when equation (13) is applied to one-dimensional flows) even if $U_{D}$ (which is an average value over the porous layer, in the direction perpendicular to surface) is not zero.

A non-uniform grid net with a higher concentration of nodal points near the interface of the vapor film and the permeable surface covers the computational
Table 1. Influence of grid size on the overall levitation pressure force $F / F_{\text {ref }}$

\begin{tabular}{llllll}
\hline$\Delta x$ & $\Delta r$ & 0.01 & 0.02 & 0.004 & 0.10 \\
\hline 0.001 & 1.02 & 1.01 & 1.00 & 0.98 \\
0.002 & 1.01 & 1.00 & 0.99 & 0.97 \\
0.004 & 0.99 & 0.98 & 0.98 & 0.96 \\
0.008 & 0.93 & 0.93 & 0.92 & 0.91 \\
\hline
\end{tabular}

$K / \alpha^{2}=10^{-20} \mathrm{~m}^{2}, \delta=1.564 \times 10^{-4} \mathrm{~m} . F_{\text {ref }}=1.983 \times 10^{-3}$ $\mathrm{N}$ determined with $\Delta r=0.02$ and $\Delta x=0.002$.

domain. A typical net has $101 \times 151\left(N_{r} \times N_{x}\right)$ nodal points. The standard convergence test (using progressively smaller grid sizes) is performed, with the conventional compromise between computational economy and accuracy. For example, the variation of the overall pressure force at the base of the droplet for evaporation over an impermeable surface $\left(K=10^{-20}\right.$ $\mathrm{m}^{2}$ ), shown in Table 1 , is within $1 \%$ when the grid spacings change from 0.01 to 0.02 and from 0.002 to 0.004 in the $r$ - and $x$-directions, respectively. The length of the computational domain, $L$, is chosen to be equal to $2 R_{0}$ and $l$ is the height of the domain above the base of the droplet (as shown in Fig. 1). For longer values of $L$ and $l$, no changes are found. The computations were performed on an IBM 3090 mainframe utilizing the vector facilities.

The solution for the stream function converges when the difference between the normalized value of $\psi$ between any two successive iterations is less than $10^{-3}$. The convergence criterion adopted for the vorticity is such that the value for the normalized difference between any two successive computations of the maximum $\omega$ in the computational domain is less than $10^{-5}$.

The algorithm is validated by computing the flow in the gap for the case where $K \rightarrow 0$, i.e. imposing the no-slip condition, and for $K=10^{-20} \mathrm{~m}^{2}$ using the Beavers-Joseph boundary condition. In each case the results are identical to the analytical results for impermeable surfaces.

\section{RESULTS AND DISCUSSION}

For evaporation over a permeable surface, it is expected that some of the vapor will penetrate the porous layer, reducing the vapor film thickness and increasing the heat transfer and evaporation rates. A new quasi-steady film thickness will then be reached where the upward pressure force exerted at the base of the droplet balances the weight. For the results presented below, the fluid is methanol and the physical properties are $\rho=0.74 \mathrm{~kg} \mathrm{~m}^{-3}, \rho_{1}=773 \mathrm{~kg} \mathrm{~m}^{-3}, \mu=$ $204 \times 10^{-1} \mathrm{~kg} \mathrm{~m}^{-1} \mathrm{~s}^{-1}, k=3.33 \times 10^{-2} \mathrm{~W} \mathrm{~m}^{-1} \mathrm{~K}^{-1}$, $h_{\mathrm{lv}}=1107.7 \mathrm{~kJ} \mathrm{~kg}^{-1}, T_{\text {sat }}=337.7 \mathrm{~K}, T_{\mathrm{p}}=650 \mathrm{~K}$, and $R_{0}=5 \times 10^{-3} \mathrm{~m}$.

Figures 2 and 3 show the radial and vertical velocity profiles (at $R=4 \mathrm{~mm}$ from the center line) for three 


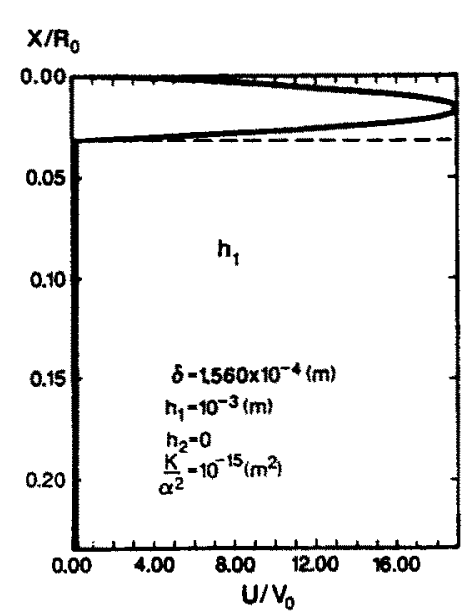

(a)

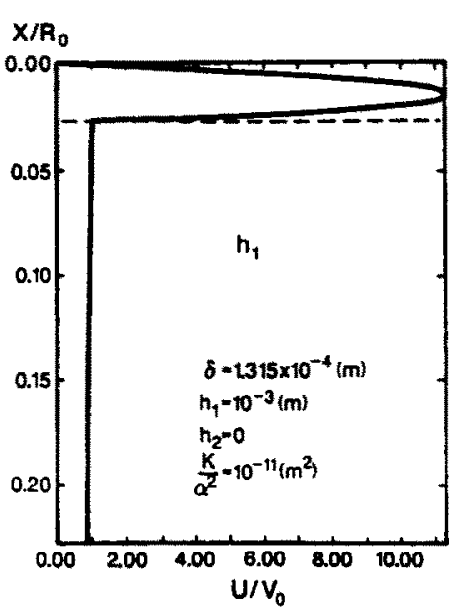

(b)

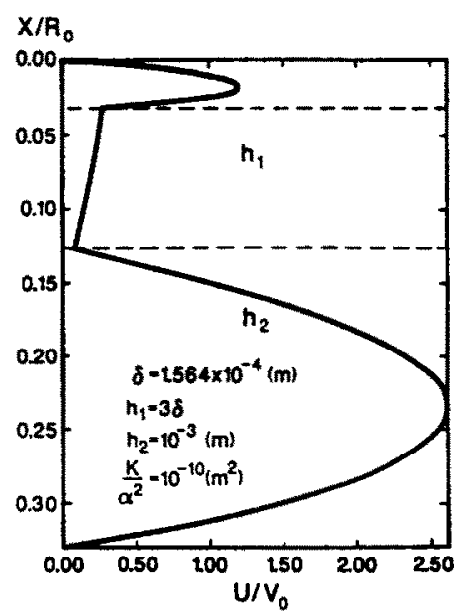

(c)

FIG. 2. Radial velocity profiles in the vapor film and the porous layer (at $R=4 \mathrm{~mm}$ from the center line) for: (a) $K / \alpha^{2}=10^{-13} \mathrm{~m}^{2}, h_{1}=10^{-3} \mathrm{~m}, h_{2}=0 ;$ (b) $K / \alpha^{2}=10^{-11} \mathrm{~m}^{2}, h_{1}=10^{-3} \mathrm{~m}, h_{2}=0:$ (c) $K / x^{2}=10^{-10}$ $\mathrm{m}^{2}, h_{1}=4.7 \times 10^{-4} \mathrm{~m}, h_{2}=10^{-3} \mathrm{~m}$.

cases: (a) $K / x^{2}=10^{-15} \mathrm{~m}^{2}, h_{1}=10^{-3} \mathrm{~m}, h_{2}=0$; (b) $K / x^{2}=10^{-11}, h_{1}=10^{-3}, h_{2}=0$; and (c) $K / x^{2}=$ $10^{-10}, h_{1}=4.7 \times 10^{-4}, h_{2}=10^{-3} \mathrm{~m}$, where the parameter $K / x^{2}$ is the permeability divided by the second power of the proportionality constant in the Beavers-Joseph interfacial model. The vapor film thickness, $\delta$, in all cases is determined from the balance of the pressure forces underneath the droplet with the weight of the droplet (equations (15) and (16)). Case (a) simulates evaporation over a nearly impermeable surface. Case (b) corresponds to a permeable upper surface $\left(K / \alpha^{2}=10^{-11} \mathrm{~m}^{2}\right)$ and an impermeable lower surface $\left(h_{2}=0\right)$. Case (c) allows for vapor penetration into the upper and lower surfaces of the porous layer (i.e. $h_{2} \neq 0$ ).
It is apparent from the velocity profiles in Figs. 2(a) and $3(a)$ that for the low permeability, $K / \alpha^{2}=10^{-15}$ $\mathrm{m}^{2}$, the vapor encounters a high resistance at the porous surface and consequently does not penetrate it. The radial velocity profile resembles that of the fully developed laminar flow between parallel plates. For the higher permeability in Figs. 2(b) and 3(b), $K / \alpha^{2}=10^{-11} \mathrm{~m}^{2}$, it is evident that the vapor penetrates into the porous layer. It is perhaps worth mentioning that the maximum radial velocity in the vapor film diminishes as the permeability of the porous surface increases. This is expected since a higher degree of vapor penetration results in a reduction of the flow rate in the vapor film, as well as the loss of momentum in the porous matrix. The reduction in the mag-

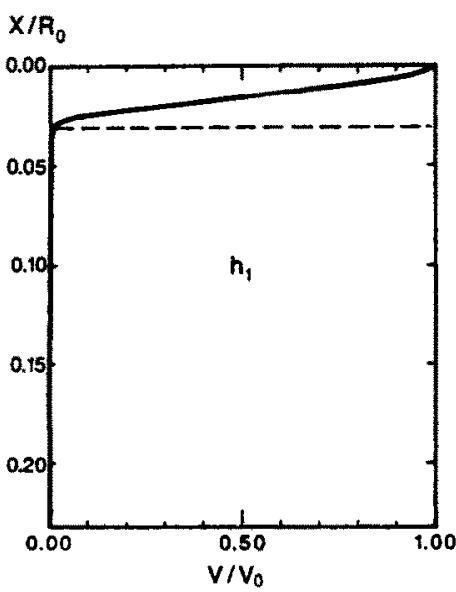

(a)

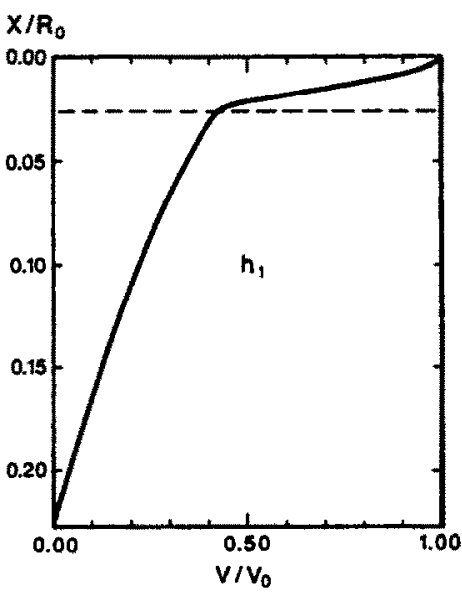

(b)

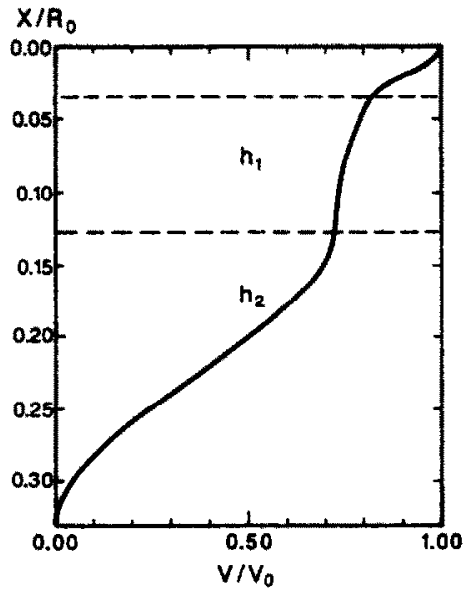

(c)

Fig. 3. Vertical velocity profiles, same conditions as in Fig. 2. 
(a)

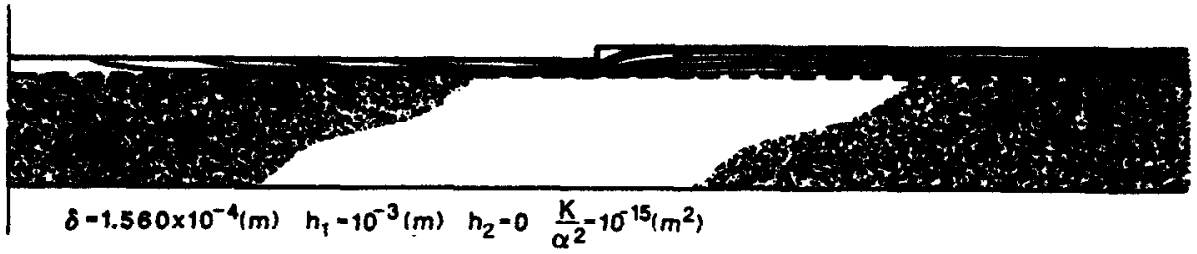

(b)

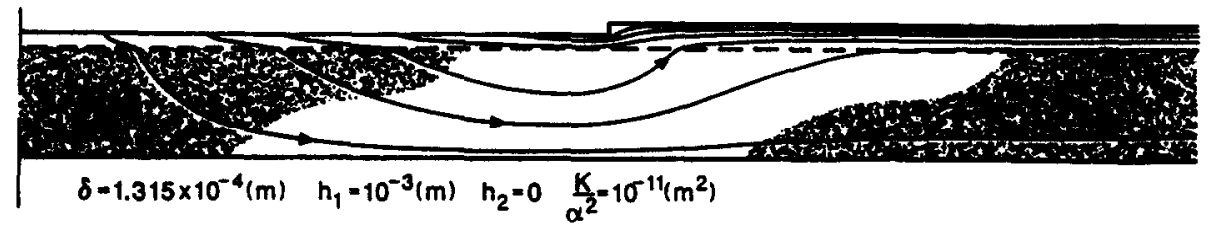

(c)

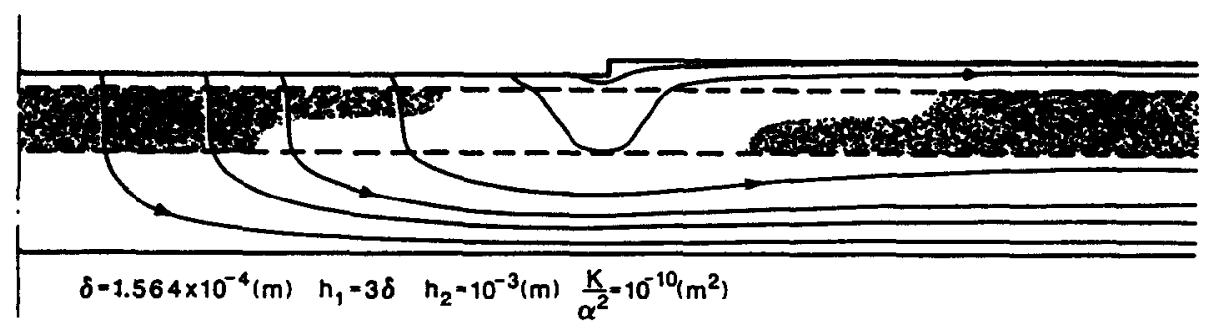

FIG. 4. Plots of streamlines for the three cases in Figs. 2 and 3.

nitude of the radial velocity is evidenced in Figs. 2(a) and (b).

Figures 2(c) and 3(c) show the penetration of the vapor through the top and bottom surfaces of the porous layer. This is illustrated in Fig. 2(c) by the two parabolic velocity profiles in the upper and lower vapor layers in contrast to the linear profile in the middle porous layer.

The effect of the permeability on the extent of vapor penetration in the porous matrix can also be inferred from the lines of constant stream function (Fig. 4). The streamlines are plotted for the values $0,0.01$, $0.05,0.10,0.20,0.35,0.45$, and 0.50 , from left to right respectively, for the three indicated cases of evaporation. For $K / \alpha^{2}=10^{-15} \mathrm{~m}^{2}$, the streamlines all lie in the vapor film (Fig. $4(\mathrm{a})$ ). For $K / x^{2}=10^{-11} \mathrm{~m}^{2}$, the streamlines with values higher than 0.35 lie in the vapor film, while the lower values of $\psi$ penetrate the porous layer. However, the vapor tends to change

t The one-dimensional momentum equations for flow in the vapor film and porous layer, given as $\mathrm{d}^{2} U / \mathrm{d} X^{2}=$ $(l / \mu)(\mathrm{d} p / \mathrm{d} R)$ and $\mathrm{d}^{2} U_{\mathrm{D}} / \mathrm{d} X^{2}=\left(\varepsilon / \mu_{\mathrm{ef}}\right)(\mathrm{d} p / \mathrm{d} R)+(\varepsilon / K) U_{\mathrm{D}}$, are solved for the boundary conditions $U(0)=0, U(\delta)=$ $U_{\mathrm{D}}(\delta), \mu(\mathrm{d} U(\delta) / \mathrm{d} X)=\mu_{\mathrm{en}}\left(\mathrm{d} U_{\mathrm{D}}(\delta) / \mathrm{d} X\right)$ and $U_{\mathrm{D}}\left(\delta+h_{1}\right)=0$.

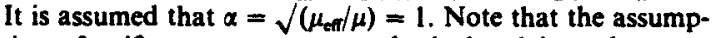
tion of uniform pressure across both the plain and porous layers results in a flow that remains within the porous layer instead of returning to the plain layer. direction and flow out of the porous matrix due to the high resistance it encounters. In fact, almost all of the vapor flows out of the porous medium through the upper surface, as can be seen in Fig. 4(b), while a small portion $(\psi=0.01)$ exits through the side of the porous matrix. When the lower surface is permeable and the porous layer is thin (case (c)), some of the penetrated vapor will escape through the bottom surface of the matrix (Fig. 4(c)).

Figure 5(a) shows the variation of initial film thickness, normalized by the radius of the droplet, with the parameter, $K / \alpha^{2}$. Initial film thickness, $\delta$, is determined iteratively from the balance of the surface integral of pressure forces exerted at the base of the droplet with the weight of the droplet (equation (16)). As expected and obtained by numerical integrations, no pressure gradient exists beyond the radius of the droplet. For comparison, the initial levitation heights obtained from the one-dimensional analysist given by Avedisian and Koplik [1] are also presented. The lines through the data are curves of best fit. It is apparent from these results that the two models predict no major vapor penetration through the surface for values of $K / \alpha^{2}$ ( $\alpha=1$ for the one-dimensional model) less than $10^{-12} \mathrm{~m}^{2}$. Unlike the one-dimensional model, the axisymmetric analysis does not predict the collapse of the droplet (as evidenced by the asymptotic behavior of $\delta / R_{0}$ for high permeabilities). Similar 

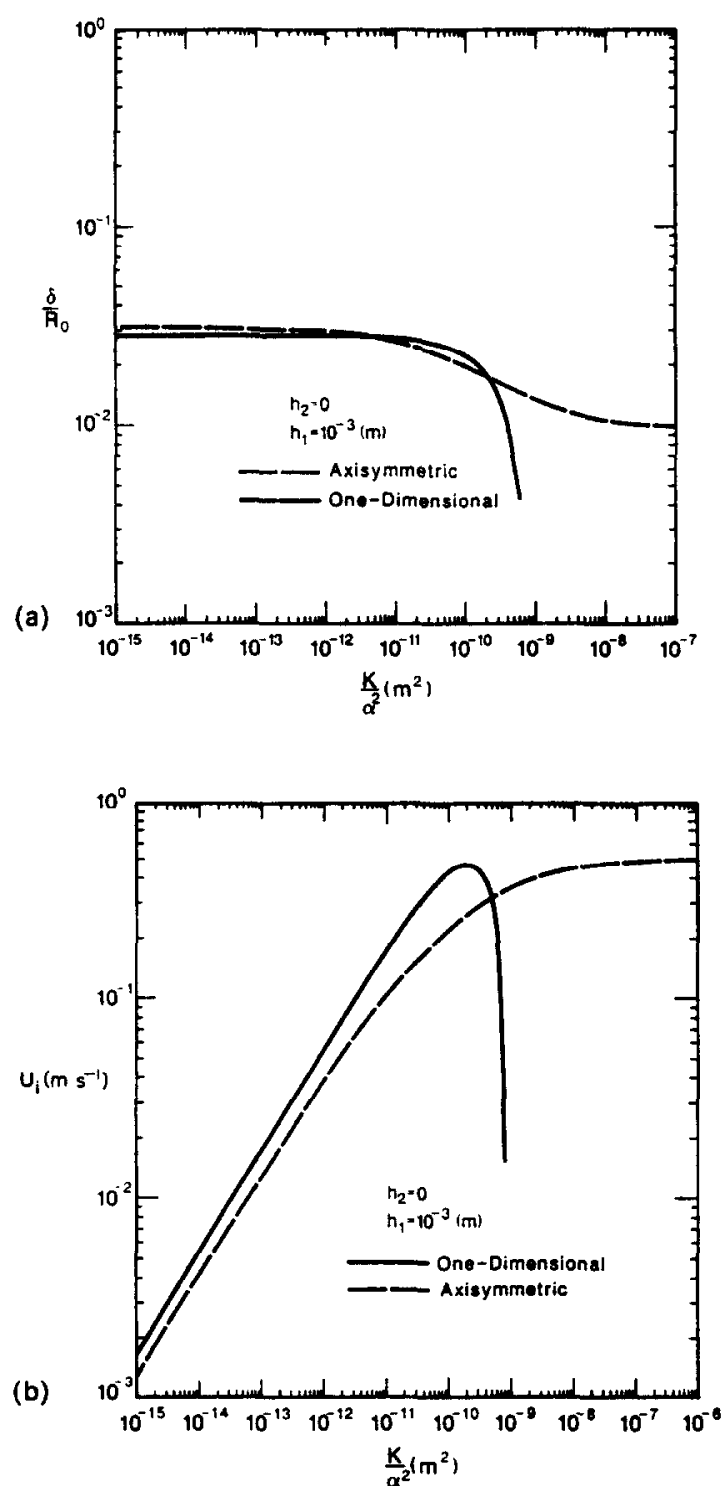

FIG. 5. Effects of $K / x^{2}$ on the normalized film thickness and the interface velocity for $h_{1}=10^{-3} \mathrm{~m}$ and $h_{2}=0$ (onedimensional and axisymmetric).

asymptotic behavior is observed for the interface velocity predicted by the axisymmetric model; this behavior contrasts with that of the one-dimensional model, which predicts a sharp drop in the radial velocity for permeabilities higher than $10^{-10} \mathrm{~m}^{2}$ (Fig. $5(b))$. An explanation of this behavior can be offered based on the limitations of the Beavers-Joseph interfacial condition. For values of $K / \alpha^{2}$ equal to or greater than $10^{-10} \mathrm{~m}^{2}$, the average size of the individual pores within the porous matrix exceeds the thickness of the vapor layer, thus the assumption of rectilinear flow (for the one-dimensional model of Beavers and Joseph) in the film breaks down. No general relationship between the permeability and particle or pore size exists [15]. The porous media which may be used in practice, for the problem considered, will be con-
Table 2. Ratio of the vapor gap size to the particle size (using equation (18))

\begin{tabular}{lcr}
\hline$K / \alpha^{2}$ & \multicolumn{1}{c}{$d_{\mathrm{p}}$} & $\delta / d_{\mathrm{p}}$ \\
\hline $10^{-13}$ & $1.2 \times 10^{-5}$ & 13.2 \\
$10^{-12}$ & $3.8 \times 10^{-5}$ & 4.2 \\
$10^{-11}$ & $1.2 \times 10^{-4}$ & 1.3 \\
$10^{-10}$ & $3.8 \times 10^{-4}$ & 0.4 \\
$10^{-9}$ & $11.9 \times 10^{-4}$ & 0.1 \\
\hline$\delta=1.564 \times 10^{-4} \mathrm{~m}$.
\end{tabular}

solidated and 'particles' or pore size will not be uniform. However, the Carman-Kozeny equation, which is valid for non-consolidated spheres of uniform size, i.e.

$$
K=\frac{d_{\mathrm{p}}^{2} \varepsilon^{3}}{180(1-\varepsilon)^{2}}
$$

is used to illustrate the relation between the average particle size and $K / \alpha^{2}$ for $\varepsilon=0.4$. It is evident from Table 2 that for $K / \alpha^{2}=10^{-10}$ or $10^{-9} \mathrm{~m}^{2}$ the thickness of the vapor layer, $\delta$, is less than the average particle size, $d_{p}$. This suggests that the levitation height is less than the size of the individual pores.

A similar argument can be made against the validity of the one-dimensional model for values of $K / x^{2}>10^{-10} \mathrm{~m}^{2}$. In the one-dimensional analysis, the vapor flow in the porous matrix is assumed to be governed by

$$
-\frac{\mu}{K} U_{\mathrm{D}}+\mu_{\mathrm{efr}} \frac{\mathrm{d}^{2} U_{\mathrm{D}}}{\mathrm{d} X^{2}}=\frac{\mathrm{d} p_{\mathrm{D}}}{\mathrm{d} R}
$$

where $U_{\mathrm{D}}$ denotes the local mean velocity within the porous matrix and $\mu_{\text {eff }}$ is an effective viscosity, which may differ from the viscosity in the plain vapor film. The assumed boundary conditions at the interface are

$$
\begin{gathered}
U(0)=U_{\mathrm{D}}(0) \\
\mu \frac{\mathrm{d} U(0)}{\mathrm{d} X}=\mu_{\mathrm{err}} \frac{\mathrm{d} U_{\mathrm{D}}(0)}{\mathrm{d} X} .
\end{gathered}
$$

Brinkman's model (equation (19)) extends the shear stress, which is present in the plain layer, into the saturated solid matrix. This can be seen as an extension to Darcy's model, which does not include variations of shear stress in the porous matrix. Brinkman's model thus predicts the formation of a very thin boundary layer within the porous matrix, which allows for the transition of the velocity from that of the fluid ( $U_{\mathrm{i}}$ at the interface) to the Darcean velocity $\left(U_{\mathrm{D}}\right)$ below the interface (Fig. 6(a)). Figures 6(b) and (c) illustrate the velocity profiles predicted by the Beavers-Joseph interfacial model for the two conditions of $U_{\mathrm{D}}=0$ and $U_{\mathrm{D}} \neq 0$ in equation (14), respectively. Darcean velocity, $U_{D}$, in Fig. 6(c) is determined by taking the average of the radial component of velocity along the $x$-direction in the porous layer. 


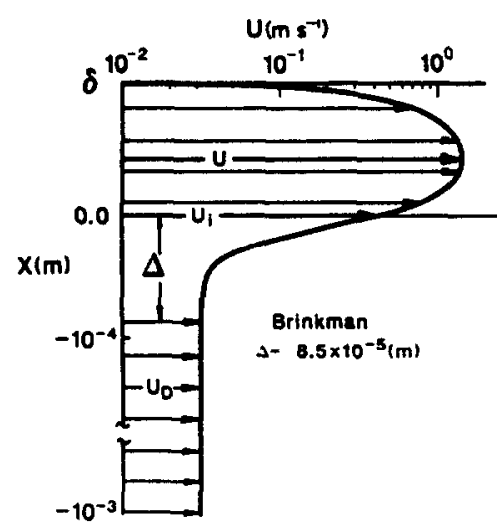

(a)

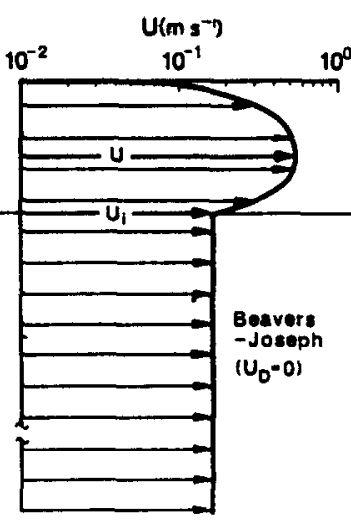

(b)

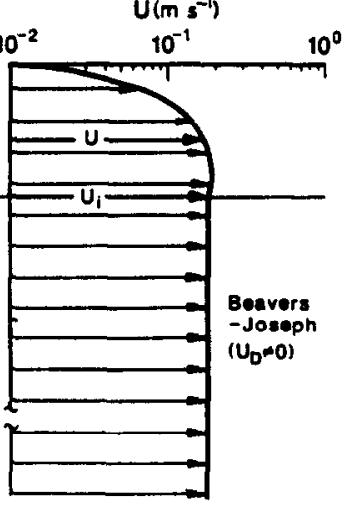

(e)

$$
\begin{aligned}
& \delta=1.1 \times 10^{-4}(\mathrm{~m}) \\
& \frac{K}{\alpha^{2}}=10^{-10}\left(\mathrm{~m}^{2}\right)
\end{aligned}
$$

FIG. 6. Radial boundary layer velocity profiles for: (a) interface velocity with no slip (Brinkman): (b) interface velocity with slip (Beavers-Joseph with $U_{D}=0$ ) ; (c) interface velocity with slip (Beavers-Joseph with $U_{\mathrm{D}} \neq 0$ ).

Setting $U_{\mathrm{D}}$ not equal to zero in equation (14) will force the flow to go through the porous layer.

The thickness of the boundary layer for one-dimensional flow can be evaluated as [8]

$$
\Delta=\sqrt{ } K \ln \left[\frac{50\left(\sigma^{2}-2\right)}{1+\sigma \sqrt{ }\left(\mu_{\mathrm{ef}} / \mu\right)}\right]^{\sqrt{ }\left(\mu_{\mathrm{er}} / \mu\right)}
$$

where $\sigma=\delta / \sqrt{ } K$. The thickness of the boundary layer as predicted by equation (22) is of the order of $10^{-5}$ $\mathrm{m}$ for a gap size of the order of $10^{-4} \mathrm{~m}$ (vapor film thickness) and a permeability of $10^{-10} \mathrm{~m}^{2}$. This implies that the thickness of the boundary layer may be less than the average particle size (depending on the relationship between the permeability and the particle size) in the porous matrix, so the application of a continuum analysis such as Brinkman's in the boundary layer produces physically unrealistic results. It should be noted that properties such as the effective viscosity, $\mu_{\text {eff }}$, or the Darcean velocity, $U_{\mathrm{D}}$, are the volume-averaged properties, i.e. averaged over a length scale larger than the individual particle or pore size. Hence, the one-dimensional model of vapor flow in the porous medium, governed by Brinkman's equation, would not be valid for the cases in which $K^{1 / 2}$ (the order of the boundary layer thickness) is smaller than the average pore size of the porous matrix. It is also worth noting that for high permeabilities, the magnitude of the surface nonuniformities will be of the order of the vapor film thickness. It is possible that the higher rate of evaporation of the droplets on ceramic surfaces, as reported by Avedisian and Koplik [1], is primarily due to surface nonuniformities protruding into the vapor film and not to the reduction of the film thickness resulting from the penetration of vapor into the porous layer.

The axisymmetric and one-dimensional models are further compared in Fig. 7. The variation of the surface integral of pressure forces is shown with respect to the permeability for three porous layers, $10^{-4}$, $10^{-3}$, and $10^{-2} \mathrm{~m}$ (the vapor film thickness is constant at $\delta=1.560 \times 10^{-4} \mathrm{~m}$, corresponding to $K / x^{2}=$ $10^{-15} \mathrm{~m}^{2}$, which is the maximum film thickness over an impermeable surface). For $K / x^{2}<10^{-13} \mathrm{~m}^{2}$, both models predict that no major penetration of vapor into the porous layer takes place, as evidenced by the balance of the pressure forces. $F$, with the weight of the droplet, $W\left(F=\dot{W}=1.98 \times 10^{-3} \mathrm{~N}\right)$. For $10^{-13}<K / \alpha^{2}<10^{-10} \mathrm{~m}^{2}$, the two models predict that the vapor penetrates through the surface and that the degree of penetration increases with higher

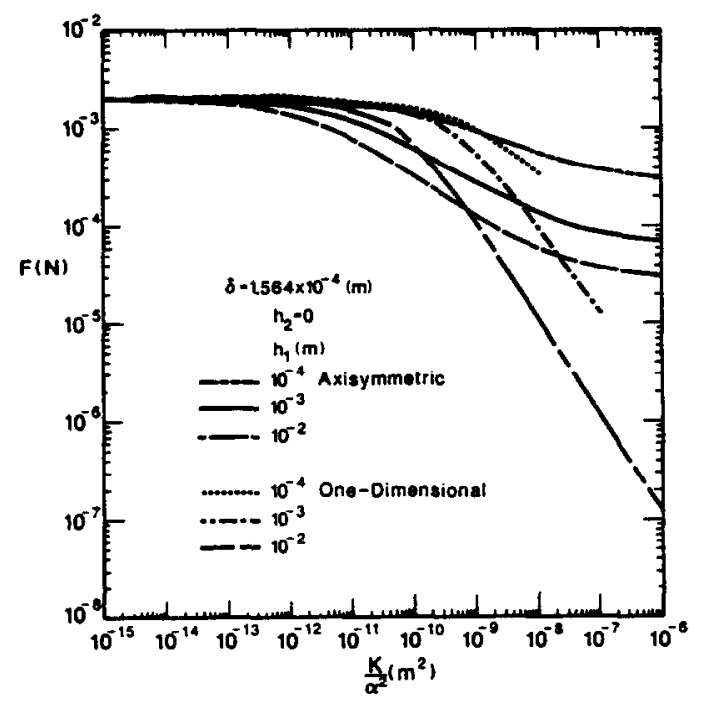

FIG. 7. Variation of $F$ with respect to $h_{1}$ and $K / x^{2}$, for $h_{2}=0$ (one-dimensional and axisymmetric). 


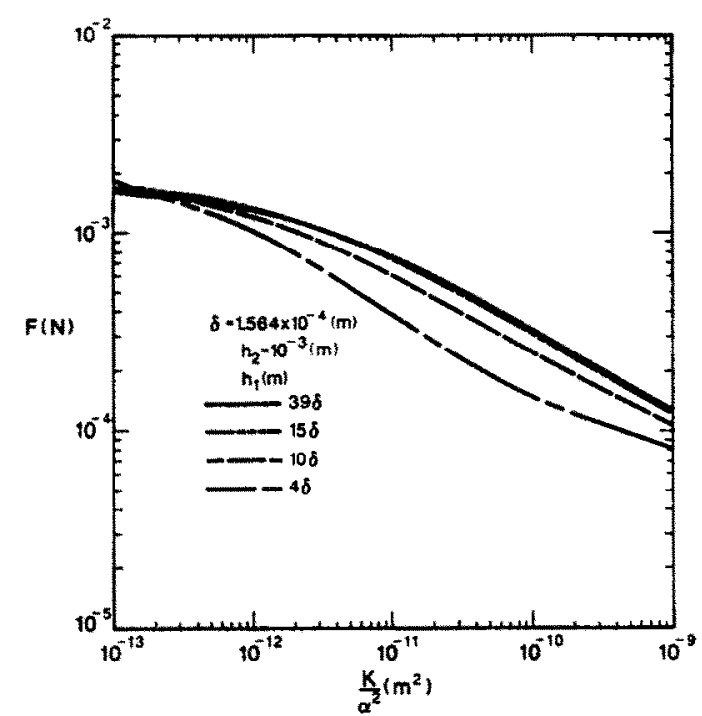

Fig. 8. Variation of $F$ with respect to $h_{1}$ and $K / \alpha^{2}$, for $h_{2}=10^{-3} \mathrm{~m}$ (axisymmetric).

permeabilities. The axisymmetric model predicts a higher degree of vapor penetration than the onedimensional model for this range of permeabilities. For $K / x^{2}>10^{-10} \mathrm{~m}^{2}$ the two models deviate from each other in their prediction of the pressure forces. This is due to the indicated limitations that the two models have at high permeabilities.

The effect of the thickness of the porous layer on the pressure forces can also be inferred from Fig. 7. For higher values of $h_{1}$, the extent of the vapor penetration into the porous medium increases, as indicated by the lower values of $F$. Increasing $h_{1}$ beyond $10^{-2} \mathrm{~m}$ seems to cause no significant change in the values of $F$, indicating that the extent of vapor penetration into the porous layer will not be greater than $0.01 \mathrm{~m}$.

The variation of the pressure forces with respect to $K / \alpha^{2}$ and $h_{1}$ for a fixed value of $h_{2}=10^{-3} \mathrm{~m}$ (i.e. the lower surface is permeable) is illustrated in Fig. $8\left(h_{1}\right.$ is given as a factor of $\delta$ in order to present the relative magnitude of the porous layer thickness with respect to the vapor layer thickness). Values of $K / \alpha^{2}<10^{-13}$ $\mathrm{m}^{2}$ are not shown since no major vapor penetration occurs through the surface and $F$ remains constant independent of $h_{1}$ and $h_{2}$. It is apparent from Fig. 8 that for values of $h_{1}>15 \delta(2.3 \mathrm{~mm}), F$ does not vary significantly. This implies that the extent of vapor penetration is approximately $2.3 \mathrm{~mm}$ (consistent with the results shown in Fig. 7). The effect of $h_{2}$ on the pressure forces is illustrated in Fig. 9. The results are plotted for a constant porous layer thickness of approximately $10 \delta(1.56 \mathrm{~mm})$ and $h_{2}$ of $10^{-4}, 10^{-3}$, $10^{-2}$, and $10^{-1} \mathrm{~m}$. Increasing $h_{2}$ beyond $10^{-2} \mathrm{~m}$ does not cause any changes in $F$. It can be inferred from Fig. 9 that as $h_{2}$ increases, more vapor escapes through the bottom surface, allowing for larger reductions in $F$. This implies that the thickness of the vapor film dimin-

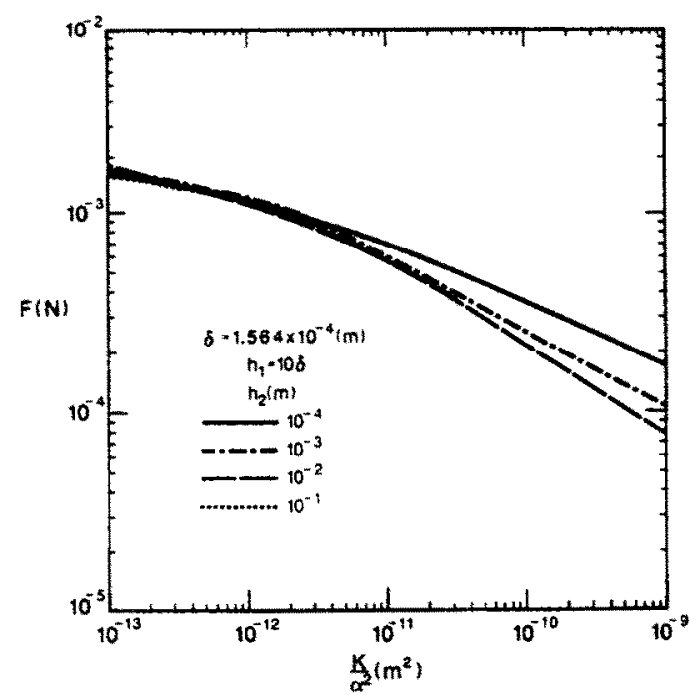

Fic. 9. Variation of $F$ with respect to $h_{2}$ and $K / \alpha^{2}$, for $h_{1}=10^{-3} \mathrm{~m}$ (axisymmetric).

ishes with increasing $h_{2}$. The upper limit of $h_{2}$ is determined to be $10 \mathrm{~mm}$, beyond which $h_{2}$ has no significant effect on the size of the gap.

\section{SUMMARY}

Assuming that it remains valid, the Beavers-Joseph boundary condition is applied to the axisymmetric flow associated with droplet levitation. The Brinkman extension, used in a previous study, is also examined. The results indicate the following.

(a) The Brinkman extension results in boundary layer thicknesses in the porous layer that are of the order of the square root of permeability ; in some cases these boundary layers are smaller than the length scale associated with the elementary representative volume.

(b) Because of the small gap sizes (for most common fluids) the surface roughness, which is also of the order of the square root of the permeability, plays a significant role and tends to reduce the average gap size.

(c) The Beavers-Joseph boundary condition appears to give physically reasonable results for the range in which it is valid.

(d) A significant increase in the evaporation is possible by using a relatively thick porous layer (compared to the gap size) or by using a thin porous layer bounded below by a plain layer.

\section{REFERENCES}

1. C. T. Avedisian and J. Koplik, Leidenfrost boiling of methanol droplets on hot porous/ceramic surfaces, Int. J. Heat Mass Transfer 30, 379-393 (1987).

2. M. Kaviany, Boundary-layer treatment of forced convection heat transfer from a seminfinite flat plate $\mathrm{em}$ bedded in porous media, $J$. Heat Transfer 109, 345349 (1987).

3. M. Kaviany, Gradient destruction in flow through a rigid matrix, J. Fluid Mech. 165, 221-230 (1986). 
4. R. E. Larson and J. J. L. Hingdon, Microscopic flow near the surface of two-dimensional porous media. Part 1. Axial flow, J. Fluid Mech. 166, 449-472 (1986).

S. R. E. Larson and J. J. L. Hingdon, Microscopic flow near the surface of two-dimensional porous media. Part 2. Transverse flow, J. Fluid Mech. 178, 119-136 (1987).

6. M. S. Moaveni-Sabet, Momentum transfer rates of a Newtonian fluid flowing above and through a porous medium, Ph.D. Thesis, Colorado State University (1987).

7. G. S. Beavers and D. D. Joseph, Boundary conditions at a naturally permeable wall, $J$. Fluid Mech. 30, 197207 (1967).

8. G. Neale and W. Nader. Practical significance of Brinkman's extension of Darcy's law: coupled parallel flows within a channel and a bounding medium, Can.J. Chem. Engng 52, 475-478 (1974).

9. K. Vafai and R. Thiyagaraja, Analysis of flow and heat transfer at the interface region of a porous medium, Int. J. Heat Mass Transfer 30, 1391-1404 (1987).

10. D. A. Nield, Onset of convection in a fluid layer overlying a layer of a porous medium, J. Fluid Mech. 81, Part III, 513-522 (1977).

I1. D. A. Nield, The boundary correction for the RayleighDarcy problem: limitations of the Brinkman equation, J. Fluid Mech. 128, 37-46 (1983).
12. D. A. Nield. Corrigendum to: The boundary correction for the Rayleigh-Darcy problem: limitations of the Brinkman equation, J. Fluid Mech. 150, 503 (1985).

13. P. G. Saffman. On the boundary condition at the surface of a porous medium, Stud. Appl. Math. 50, 93 (1971).

14. Y. Jaluria and K. E. Torrance, Computational Heat Transfer. Hemisphere. Washington. DC (1986).

15. F. A. L. Dullien. Porous Media: Fluid Transport and Structure. Academic Press, New York (1979).

\section{APPENDIX}

As was indicated. inclusion of $U_{\mathrm{D}}$ in the interfacial boundary condition (equation (14)) implies the presence of a pressure gradient in the porous layer, which is the force driving $U_{\mathrm{D}}$. This is illustrated in Fig. Al, which presents the flow (streamlines) for the case previously shown in Fig. 4(c), except that $U_{D}$ is not set to zero. Note that the flow continues through the porous layer, even at large distances from the edge of the droplet. This flow is not realistic because the flow is expected to favor a return to the gap rather than continuing in the porous layer where the resistance is significantly larger. Based on this. Darcy's velocity was not included in the boundary condition.

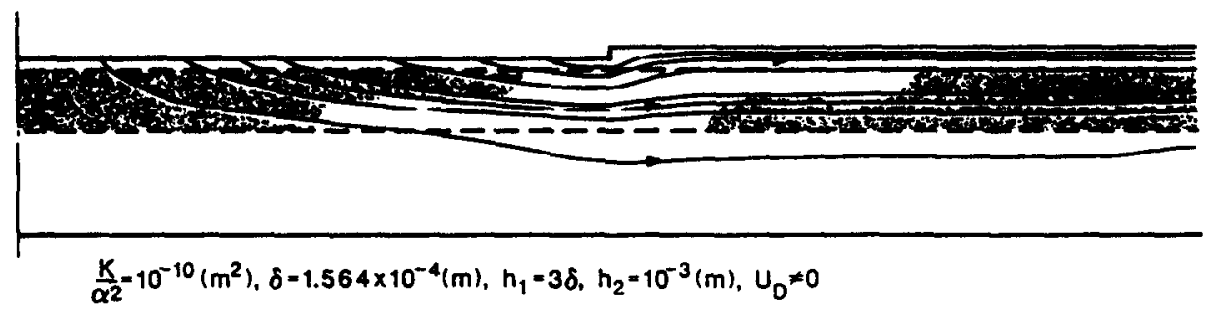

FIG. Al. Same conditions as in Fig. 4(c) except $U_{D} \neq 0$.

\title{
ANALYSE DE LA LEVITATION DES GOUTTELETTES DE LIQUIDE SATURE SUR DES SURFACES PERMEABLES
}

\begin{abstract}
Résumé-L'évaporation d'une gouttelette de liquide saturé et la lévitation sur une surface solide due à la pression induite par la quantité de mouvement dans le film de vapeur sont étudiées en résolvant numériquement l'équation axisymétrique. Une analyse monodimensionnelle antérieure montre que la distance entre la surface solide et la gouttelette décroît quand la vapeur peut pénétrer dans le solide. Pour les couches poreuses, une analyse monodimensionnelle qui est basée sur l'extension selon Brinkman de la loi de Darcy prédit des épaisseurs de quantité de mouvement, pour la couche limite, de l'ordre de la racine carrée de la perméabilité. Dans des solides poreux typiques, cette épaisseur est plus petite que le pore ou la taille de la particule. Dans la présente étude la condition limite semi-empirique de Beavers-Joseph est utilisée à la place du modèle monodimensionnel. La réduction de la séparation par variation de la perméabilité et de l'épaisseur de la couche poreuse est déterminée dans des domaines oủ cette condition limite est valable. La faiblesse du modèle pour prédíre le collapsus de la gouttelette est due à la défaillance de la condition interfaciale de Beavers-Joseph pour les fortes perméabilités. Ce modèle monodimensionnel, l'extension de Brinkman prédisent une croissance exagérément rapide de la distance quand la perméabilité est accrue jusqu'à une certaine valeur, à cause de l'hypothèse de gradients de pression égaux dans les couches poreuses.
\end{abstract}




\title{
UNTERSUCHUNG DER SCHWEBUNG VON GESÄTTIGTEN FLUUSSIGKEITSTROPFEN AUF DURCHLÄSSIGEN OBERFLÄCHEN
}

Zusammenfassung - Es wird die stationäre Verdampfung eines gesättigten Flüssigkeitstropfens und dessen Schwebung über einer festen Oberfläche aufgrund des vom Impuls induzierten Druckes im Dampffilm untersucht. Dazu werden die Erhaltungsgleichungen in achsensymmetrischer Darstellung numerisch gelöst. Eine frühere eindimensionale Analyse zeigt, daB der Abstand (Spalt) zwischen der festen Oberfläche und dem Tropfen abnimmt, wenn der Dampf den Festkörper durchdringen kann. Für poröse Schichten werden mit der Erweiterung nach Brinkman im Darcy-Gesetz Impulsgrenzschichtdicken in der Größenordnung der Wurzel der Permeabilitāt berechnet. In dieser Untersuchung wird die halb-empirische Randbedingung von Beavers-Joseph für die Grenzfläche der glatten und der durchlässigen Schicht verwendet. Die Spaltverminderung wurde in Abhängigkeit von der Permeabilität und der Dicke der porösen Schicht sowie der Dampfabströmung durch die poröse Schicht bestimmt. Für die Schlupfgeschwindigkeit und die SpaltgröBe wird ein asymptotischer Wert berechnet, wenn die Permeabilität über einen bestimmten Wert ansteigt. Das Versagen des Modells zur Berechnung des Tropfenzusammenbruchs ist mit der Ungülugkeit der Randbedingung von Beavers-Joseph für große Permeabilitäten verbunden. Für praktische Anwendungen jedoch wird erwartet, daß die Oberflächenrauhigkeit überwiegt, wenn die Spaltgröße den Wert Null erreicht.

\section{АНАЛИЗ ЛЕВИТАЦИИ НАСЫЩЕННЫХ КАПЕЛЬ ЖИДКОСТИ НА ПРОНИЦАЕМЫХ ПOBEPXHOCTЯX}

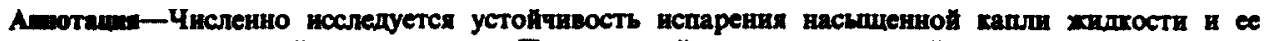

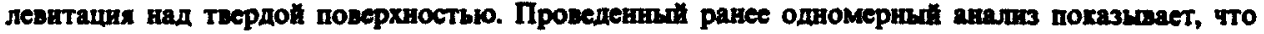
расстохние (промехутох) мехду твердой поверхностью и каплећ̆ уменьшается ири пронпиовении пара в твердое тело. Длх порнстых слоев одномерный анализ захона Дарси на оснохе подхода Бринкмана позволяет определить толиину потери импульса ках величину порядка квалратного корня из значения проницаемости. В титиных пористых твердых телах эта толшина меньше размера пор или частиц. В настопием носледовяин мместо одномерной модели исползуется

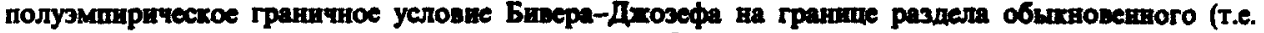

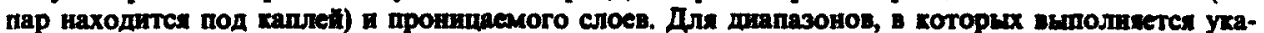
занное граничне условие, определено уменишение размера промехутков с пзмененвем пронищае-

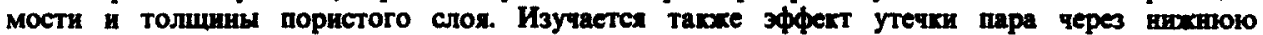
поверхность порнстого слоя. Данная модель позволяет рассчнтать аснмттотнческое значение сто-

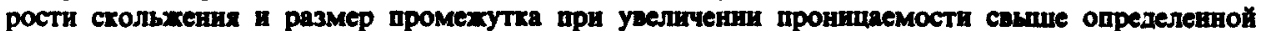

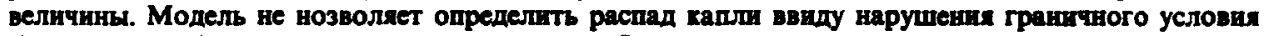
Бивера-Дхозефа при висохой пронпиаемости. Однахо надо иметь в виду, тто на праттике шероховатость поверхности будет преобладать в случае приблихении размера промехутка к нуль. C друтой стороны, одномерная модел, резработаннал Бринхманом, дает нереально быстрое уменьшение промехутха при уреличени пронпиемости свипе определенио веничины в предполохении равенства градиентов давления в пористом и обыкновенном слоях.
\end{abstract}

